DOI: $10.5216 /$ cab.v13i4.19468

\title{
COMPARAÇÃO DA FIBRA EM DETERGENTE NEUTRO DE FORRAGENS: DESEMPENHO, DIGESTIBILIDADE E PARÂMETROS RUMINAIS
}

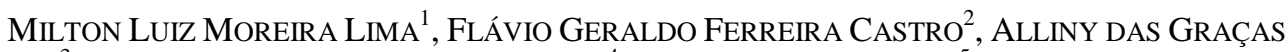 \\ AMARAL $^{3}$, EDUARDO RODRIGUES DE CARVALHO ${ }^{4}$, LUIZ GUSTAVO NUSSIO ${ }^{5}$, WILSON ROBERTO SOARES \\ MATTOS
}

\author{
${ }^{1}$ Professor Doutor da Universidade Federal de Goiás, Goiânia, GO, Brasil \\ ${ }^{2}$ Médico Veterinário Doutor da Agrocria Coméricio e Indústria LTDA, Goiânia, GO, Brasil \\ ${ }^{3}$ Professora Doutora da Universidade Estadual de Goiás, São Luis de Montes Belos, GO, Brasil. \\ ${ }^{4}$ Professor Doutor do Instituto Federal Goiano, Iporá, GO, Brasil - eduardo.carvalho@ifgoiano.edu.br \\ ${ }^{5}$ Professores Doutores da Escola Superior de Agricultura Luiz de Queiroz, USP, Piracicaba, SP, Brasil
}

\begin{abstract}
Objetivou-se determinar os efeitos da substituição parcial da fibra em detergente neutro (FDN) da silagem de milho pela FDN da cana-de-açúcar in natura ou cana-de-açúcar tratada com $\mathrm{NaOH}$ ou feno de alfafa, sobre o desempenho, digestibilidade e parâmetros ruminais de vacas Holandesas em lactação, canuladas no rúmen e no duodeno, distribuídas em um delineamento Quadrado Latino $5 \times 5$. Utilizaram-se duas dietas controle, uma com baixo (controle negativo; SMB) e outra com alto (controle positivo; SMA) teor de FDN de forragem (SMB com 14\% e SMA com $22 \%$ de FDN da silagem de milho). Três rações experimentais foram balanceadas para conter $14 \%$

de FDN da silagem de milho e 8\% de FDN da cana-deaçúcar in natura (CAN) ou cana-de-açúcar tratada com $\mathrm{NaOH}$ (CAS) ou feno de alfafa (FA). O consumo de matéria seca (MS), a produção e composição do leite não foram afetados $(\mathrm{P}>0,05)$ pelas dietas. Houve resposta $(\mathrm{P}<0,05)$ da concentração e fonte da FDN da forragem sobre o pH ruminal, proporções molares de ácidos graxos de cadeia curta (AGCC) e relação acetato:propionato. Os resultados indicam a viabilidade da substituição de $8 \%$ de FDN da silagem de milho pela FDN da cana-de-açúcar in natura na alimentação de vacas leiteiras com produção de $18,2 \mathrm{~kg}$ de leite/dia.
\end{abstract}

PALAVRAS-CHAVE: cana-de-açúcar; feno de alfafa; gordura; hidrólise; silagem de milho.

\section{COMPARISON OF FORAGE NEUTRAL DETERGENT FIBER: PERFORMANCE, DIGESTIBILITY AND RUMEN PARAMETERS}

\section{ABSTRACT}

The objective of this study was to determine the effects of the partial replacement of corn silage NDF by sugarcane $\mathrm{NDF}$ or sugarcane treated with $\mathrm{NaOH} \mathrm{NDF}$ or alfalfa hay NDF, on performance, digestibility and rumen parameters of lactating Holstein cows, cannulated in the rumen and duodenum, which were assigned in a $5 \times 5$ Latin Squared design. Two control diets were utilized, one with low (negative control; SMB) and another one with high (positive control; SMA) forage NDF concentration (SMB containing $14 \%$ and SMA containing $22 \%$ forage NDF from corn silage). Three experimental rations were balanced to contain $14 \%$ corn silage NDF and $8 \%$ sugarcane NDF $(\mathrm{CAN})$ or sugar-cane treated with $\mathrm{NaOH}$ NDF (CAS) or alfalfa hay NDF (FA). Feed intake, milk production and milk composition were not affected $(\mathrm{P}>0.05)$ by diets. There was response $(\mathrm{P}<0.05)$ of the concentration and source of forage NDF on rumen $\mathrm{pH}$, molar proportions of short-chain fatty acids, and acetate:propionate ratio. The data of this experiment indicate the viability of replacing $8 \%$ corn silage NDF by sugarcane NDF fed to dairy cows with milk yield of 18.2 $\mathrm{kg} / \mathrm{day}$

KEYWORDS: alfalfa hay; corn silage; fat; hydrolysis; sugarcane. 


\section{INTRODUÇÃO}

A fibra em detergente neutro (FDN) é um componente essencial da dieta de vacas em lactação, sendo necessária para a obtenção do máximo consumo de matéria seca (MS) e energia, o estímulo à atividade de mastigação e secreção de saliva, e ainda para manter a concentração de gordura no leite em níveis adequados. Assim, dietas para vacas em lactação devem conter uma concentração mínima de FDN para manter a função ruminal normal e evitar a ocorrência de distúrbios metabólicos. Por outro lado, a FDN representa a fração de carboidratos dos alimentos de digestão lenta e variável e, quando incluída acima de determinados limites definidos pelo potencial de produção animal, pode limitar tanto o consumo de MS quanto o desempenho (MERTENS, 1997). O NRC (2001) sugere que dietas para vacas em lactação devem conter no mínimo 25\% de FDN do total da MS da ração, sendo $75 \%$ suprido por forragens.

No entanto, os limites de FDN nas rações de bovinos leiteiros são definidos não apenas por concentrações mínimas e máximas, mas também por atributos físicos, químicos e biológicos dos alimentos, que podem alterar o desempenho, digestão e o teor de gordura no leite. Assim, dois conceitos foram desenvolvidos para o balanceamento de FDN, considerando-se esses atributos complementares. Define-se por fibra efetiva (FDNe) a capacidade da FDN de um alimento substituir a FDN da forragem (FDNf) sem alterar a concentração de gordura no leite, sendo, portanto, um atributo biológico. Por outro lado, a fibra fisicamente efetiva (FDNfe) consiste na FDN de um determinado alimento que tem o potencial de estimular a atividade mastigatória, ou seja, um atributo exclusivamente físico, o qual está relacionado ao tamanho da partícula do alimento (MERTENS, 1997). Dessa forma, variações na digestibilidade da FDN e no tamanho da partícula dos alimentos alteram os valores de FDNe e FDNfe, respectivamente, influenciando o teor de gordura no leite e/ou a atividade de mastigação.

A importância da FDNe de plantas forrageiras sobre o teor de gordura no leite vem sendo intensamente avaliada, porém os resultados encontrados na literatura nem sempre são consistentes (MOONEY \& ALLEN, 1997; CLARK \& ARMENTANO, 1999; BAL et al., 2000; SOITA et al., 2000; SCHWAB et al., 2002), sendo que, na maioria desses estudos, não houve resposta da FDNe sobre o teor de gordura no leite (MOONEY \&
ALLEN, 1997; BAL et al., 2000; SOITA et al., 2000; SCHWAB et al., 2002).

A silagem de milho e a cana-de-açúcar representam duas das principais fontes de forragens para a alimentação de bovinos leiteiros durante o período de estiagem no Brasil. A elevada produtividade, baixo custo por unidade de MS e manutenção do valor nutritivo por períodos prolongados são argumentos frequentemente utilizados para justificar o emprego da cana-deaçúcar na alimentação de bovinos leiteiros; entretanto, a substituição parcial ou total da silagem de milho pela cana-de-açúcar resulta em decréscimo no consumo de MS e na produção de leite (CORREA et al., 2000; MAGALHÃES et al., 2004; MENDONÇA et al., 2004; COSTA et al., 2005). Ademais, na compilação de dados de seis experimentos com delineamento em Quadrado Latino $4 \times 4$, comparando-se a alimentação exclusiva da silagem de milho ou cana-de-açúcar como fonte de forragem, verificou-se redução no consumo de MS e queda na produção de leite (OLIVEIRA et al., 2011). Um dos fatores dessa redução está na maior lignificação da parede celular da cana-de-açúcar, fazendo com que sua digestibilidade da FDN seja menor, comparada à da silagem de milho (PEREIRA et al., 2000; CAMPOS et al., 2001; MAGALHÃES et al., 2006). A avaliação da digestibilidade in vitro $\mathrm{e}$ in situ da FDN de 13 forragens demonstrou que o aumento de uma unidade percentual na digestibilidade dos carboidratos fibrosos resultou em aumento de $0,17 \mathrm{~kg} /$ dia no consumo de MS e 0,25 $\mathrm{kg} / \mathrm{dia}$ na produção de leite (OBA \& ALLEN, 1999).

A restrição do consumo de MS imposta pela menor digestibilidade da FDN da cana-de-açúcar pode ser mitigada por meio de tratamentos químicos com álcalis $(\mathrm{NaOH})$. Tais tratamentos rompem as ligações entre a lignina e os polissacarídeos da parede celular, disponibilizando esses carboidratos fibrosos para fermentação realizada pelos microrganismos do rúmen (KLOPFENSTEIN \& OWEN, 1981). Além disso, a composição da parede celular se altera, pois a maior parte da hemicelulose torna-se solúvel durante o tratamento (BERGER et al., 1979), sendo que parece não existir estudos na literatura que avaliaram a FDN da cana-de-açúcar in natura comparada à cana-de-açúcar tratada com $\mathrm{NaOH}$, tornando o presente trabalho inédito nesse aspecto.

Nesse contexto, objetivou-se neste estudo determinar os efeitos da substituição parcial da FDN da silagem de milho pela FDN da cana-de-açúcar in natura $(\mathrm{CAN})$ ou tratada com $\mathrm{NaOH}(\mathrm{CAS})$ sobre o 
desempenho, manutenção do teor de gordura do leite, digestibilidade de nutrientes e parâmetros ruminais de vacas em lactação. Como o feno de alfafa vem sendo frequentemente utilizado como referência na avaliação da FDN de forragens, incluiu-se um tratamento adicional com feno de alfafa (FA) para comparação com a FDN das demais forragens (silagem de milho, cana-de-açúcar in natura e tratada com $\mathrm{NaOH}$ ).

\section{MATERIAL E MÉTODOS}

Utilizaram-se neste estudo cinco vacas Holandesas canuladas no rúmen e no duodeno, que, ao início desta pesquisa, encontravam-se no terço médio da lactação, distribuídas em um delineamento Quadrado Latino $5 \times 5$. As vacas foram alojadas em baias individuais, equipadas com comedouro e bebedouro, localizadas no galpão para ensaios metabólicos do Departamento de Zootecnia da Escola Superior de Agricultura Luiz de Queiroz (ESALQ/USP, Piracicaba - SP).

Silagem de milho, cana-de-açúcar in natura, cana-de-açúcar tratada com $\mathrm{NaOH}$ e feno de alfafa foram usados como forragens no balanceamento das cinco dietas experimentais (Tabela 1). Formularamse as dietas para vacas em lactação, com $570 \mathrm{~kg}$ de peso vivo, produzindo $20 \mathrm{~kg}$ de leite/dia com 3,5\% de gordura e $3,3 \%$ de proteína, conforme recomendações do NRC (2001). Balancearam-se as dietas visando-se obter níveis equivalentes de nutrientes, exceto para concentrações e fontes de FDNf, que foram alteradas de acordo com os tratamentos propostos.

Balanceou-se a dieta com baixa porcentagem de FDNf (SMB) para conter $14 \%$ de FDNf proveniente da silagem de milho e $22 \%$ de FDN total, e a dieta com alta porcentagem de FDNf (SMA) para conter $22 \%$ de FDNf proveniente da silagem de milho e $28 \%$ de FDN total. Essas dietas representaram, respectivamente, os controles negativo (SMB) e positivo (SMA), sendo comparadas com três dietas que continham $14 \%$ de FDNf proveniente da silagem de milho e $8 \%$ de FDNf proveniente da cana-de-açúcar in natura (CAN), $14 \%$ de FDNf proveniente da silagem de milho e $8 \%$ de FDNf proveniente da cana-de-açúcar tratada com $\mathrm{NaOH}$ (CAS) e $14 \%$ de FDNf proveniente da silagem de milho e $8 \%$ de FDNf proveniente do feno de alfafa (FA), perfazendo $28 \%$ de FDN total nessas três rações. Realizou-se o tratamento da cana-de-açúcar durante a sua trituração, utilizando-se uma solução comercial de hidróxido de sódio ( $\mathrm{NaOH} 50 \%$ peso/volume) aplicada na proporção de $2 \%$ (peso/volume), com auxilio de um pulverizador acoplado ao equipamento de colheita (Kit hidrocana). Após a aplicação da solução com $\mathrm{NaOH}$, a cana-de-açúcar triturada permaneceu ao ar livre por um período de 24 horas para permitir a hidrólise da parede celular.

Conduziu-se o experimento por 65 dias, divididos em cinco períodos de 13 dias, sendo sete dias de adaptação às dietas experimentais e seis dias de coletas. Misturaram-se as rações manualmente duas vezes ao dia após pesagens dos alimentos, sendo fornecidas ad libitum em duas refeições diárias (06h00min e 18h00min). Pesaram-se as sobras diariamente antes da refeição da manhã e da tarde para o cálculo do consumo de MS e ajuste de sobras para $10 \%$ da quantidade oferecida, a fim de garantir o máximo consumo voluntário de MS pelos animais. Amostraram-se as dietas do oitavo ao décimo dia de cada período experimental. Congelaram-se as amostras a $-4^{\circ} \mathrm{C}$, sendo posteriormente reunidas para formar uma amostra composta por animal e tratamento ao final de cada período de coleta.

Amostras das dietas, previamente descongeladas e secas em estufa de ventilação forçada a $65^{\circ} \mathrm{C}$ durante 48 horas, foram moídas em moinho tipo Wiley dotado com peneira de $1 \mathrm{~mm}$ (Wiley Mill; Arthur H. Thomas, Philadelphia, PA) e analisadas para determinação de MS, proteína bruta $(\mathrm{PB})$, matéria mineral (MM), extrato etéreo (EE) e fibra em detergente ácido (FDA), segundo a AOAC (2000). Determinaram-se as concentrações de FDN e lignina sequencialmente de acordo com VAN SOEST et al. (1991). Utilizou-se, na determinação de FDN, sulfito de sódio e amilase termoestável (Termamyl $120 \mathrm{~L}$ ) e, na determinação de lignina, solução de $\mathrm{H} 2 \mathrm{SO} 4$ 72\%. Para determinação da FDN indigestível (FDNi) das dietas experimentais, amostras de $0,25 \mathrm{~g}$, previamente secas em estufa de ventilação forçada a $65^{\circ} \mathrm{C}$ durante 48 horas e moídas em moinho tipo Wiley dotado com peneira de $1 \mathrm{~mm}$ (Wiley Mill; Arthur H. Thomas, Philadelphia, PA), foram incubadas in vitro, por 144 horas (BERCHIELLI et al., 2000), e analisadas em seguida para determinação da FDN (VAN SOEST et al., 1991). Calculou-se a concentração de FDNpd das dietas experimentais pela seguinte equação: FDNpd $=[($ FDN - FDNi $) / F D N)] \times 100($ Tabela 1$)$. 
Tabela 1 - Composição percentual dos ingredientes e análise nutricional das dietas experimentais

\begin{tabular}{|c|c|c|c|c|c|}
\hline Item & $\mathrm{SMB}^{1}$ & $\mathrm{CAN}^{2}$ & $\mathrm{CAS}^{3}$ & FA $^{4}$ & $\mathrm{SMA}^{5}$ \\
\hline \multicolumn{6}{|l|}{ Ingredientes, \% da MS } \\
\hline Silagem de milho & 29,6 & 29,6 & 29,2 & 28,9 & 47,3 \\
\hline Milho triturado & 51,8 & 34,6 & 33,9 & 36,9 & 35,1 \\
\hline Farelo de soja & 15,6 & 18,3 & 18,0 & 5,6 & 14,4 \\
\hline Ureia & 0,5 & 0,8 & 0,8 & 0,8 & 0,7 \\
\hline Suplemento mineral/vitamínico ${ }^{6}$ & 2,5 & 2,5 & 2,5 & 2,6 & 2,5 \\
\hline Cana-de-açúcar & & 14,2 & & & \\
\hline Cana de açúcar + NaOH & & & 15,6 & & \\
\hline Feno de alfafa & & & & 25,2 & \\
\hline \multicolumn{6}{|l|}{ Análise nutricional } \\
\hline MS, \% & 54,5 & 47,1 & 47,7 & 55,0 & 43,9 \\
\hline $\mathrm{PB}, \%$ da MS & 18,2 & 18,5 & 18,0 & 17,9 & 17,8 \\
\hline $\mathrm{MM}, \%$ da MS & 6,5 & 6,5 & 6,8 & 7,6 & 7,0 \\
\hline $\mathrm{EE}, \%$ da MS & 2,3 & 2,0 & 1,9 & 2,2 & 2,4 \\
\hline $\mathrm{CNF}, \%$ da $\mathrm{MS}$ & 52,1 & 45,5 & 45,0 & 44,8 & 45,6 \\
\hline FDA, $\%$ da MS & 9,3 & 13,8 & 14,3 & 14,7 & 13,5 \\
\hline Lignina, \% da MS & 1,3 & 2,4 & 2,5 & 3,1 & 1,6 \\
\hline FDN, $\%$ da MS & 20,9 & 27,2 & 28,3 & 27,5 & 27,2 \\
\hline FDN $i, \%$ da FDN total & 3,9 & 7,5 & 7,4 & 7,9 & 6,3 \\
\hline FDNpd, \% da FDN total & 81,3 & 72,4 & 73,8 & 71,3 & 76,8 \\
\hline FDN da silagem do milho & 13,2 & 13,3 & 13,3 & 13,0 & 21,3 \\
\hline FDN da cana-de-açúcar in natura & & 8,3 & & & \\
\hline FDN da cana-de-açúcar + $\mathrm{NaOH}$ & & & 9,3 & & \\
\hline FDN feno de alfafa & & & & 9,5 & \\
\hline FDN do concentrado & 7,7 & 6,0 & 5,8 & 4,8 & 5,9 \\
\hline
\end{tabular}

${ }^{1} \mathrm{SMB}=$ silagem de milho baixa (14\% FDNf de silagem de milho e $22 \%$ FDN total)

${ }^{2} \mathrm{CAN}=$ cana-de-açúcar in natura $(14 \%$ FDNf de silagem de milho $+8 \%$ FDNf de cana-de-açúcar e $28 \%$ FDN total $)$

${ }^{3} \mathrm{CAS}=$ cana-de-açúcar $+\mathrm{NaOH}(14 \%$ FDNf de silagem de milho $+8 \%$ FDNf de cana-de-açúcar e $28 \%$ FDN total $)$

${ }^{4} \mathrm{FA}=$ feno de alfafa (14\% de FDNf de silagem de milho $+8 \%$ FDNf de feno de alfafa e $28 \%$ FDN total)

${ }^{5} \mathrm{SMA}=$ silagem de milho alta (22\% FDNf de silagem de milho e $28 \%$ FDN total)

${ }^{6}$ Composição: $22 \% \mathrm{Ca} ; 5,5 \% \mathrm{P} ; 10,5 \% \mathrm{Cl} ; 7 \% \mathrm{Na} ; 3,5 \% \mathrm{Mg} ; 2,2 \%$ S; 50 ppm Co; 450 ppm Cu; 40 ppm I; 500 ppm Fe; 1.500 ppm $\mathrm{Mn} ; 1.550 \mathrm{ppm} \mathrm{Zn;20} \mathrm{ppm} \mathrm{Se;} 90.000$ UI vit. A; 75.000 UI vit. D e 1.000 UI vit. E

$\mathrm{MS}$ = matéria seca; $\mathrm{PB}=$ proteína bruta; $\mathrm{MM}=$ matéria mineral; $\mathrm{EE}=$ extrato etéreo; $\mathrm{CNF}=$ carboidratos não fibrosos; FDA = fibra em detergente ácido; FDN = fibra em detergente neutro; FDN $i=$ fibra em detergente neutro indigestível (FDN residual após incubação in vitro por 144 horas); FDNpd = fibra em detergente neutro potencialmente digestível

$\mathrm{CNF}=100-(\% \mathrm{~PB}+\% \mathrm{MM}+\% \mathrm{EE}+\% \mathrm{FDN})$

$\mathrm{FDNpd}=[(\mathrm{FDN}-\mathrm{FDN} i) / \mathrm{FDN})] \times 100$

Determinou-se a digestibilidade das dietas no rúmen e no trato total utilizando-se a fibra em detergente ácido indigestível (FDA $i$ ) como marcador interno (BERCHIELLI et al., 2000). Coletaram-se amostras do conteúdo duodenal $( \pm 250 \mathrm{~mL})$ e fezes $( \pm 200 \mathrm{~g})$ a cada três horas, do décimo ao décimo segundo dias de cada período. Congelaram-se as amostras a $-4^{\circ} \mathrm{C}$ e, ao final de cada período, foram descongeladas, e submetidas à secagem em estufa de ventilação forçada a $65^{\circ} \mathrm{C}$, durante 48 horas, posteriormente moídas em moinho tipo Wiley dotado com peneira de $1 \mathrm{~mm}$ (Wiley Mill; Arthur $\mathrm{H}$.
Thomas, Philadelphia, PA), e armazenadas para análises posteriores. Para determinação das concentrações de FDA $i$, amostras de $0,25 \mathrm{~g}$ das dietas, conteúdo duodenal e fezes foram incubadas por 144 horas in vitro (BERCHIELLI et al., 2000) e analisadas em seguida para determinação de FDA (AOAC, 2000). Utilizou-se a equação de SCHNEIDER \& FLATT (1975) para determinação da digestilidade da MS e matéria orgânica (MO) no trato total, FDN (rúmen e trato total) e FDN potencialmente digestível (FDNpd) no rúmen e trato total: 
Digestibilidade da MS (trato total):

$$
\operatorname{Dig}=100-100 \times \frac{\text { Indicador no alimento }(\% \mathrm{MS})}{\text { Indicador nas fezes }(\% \mathrm{MS})}
$$

Digestibilidade da FDN e FDNpd (rúmen):

$$
\operatorname{Dig}=100-100 \times \frac{\text { Indicador no alimento }(\% \mathrm{MS}) \times \% \text { do nutriente no duoedeno }(\% \mathrm{MS})}{\text { Indicador no duodeno }(\% \mathrm{MS}) \times \% \text { do nutriente no alimento }(\% \mathrm{MS})}
$$

Digestibilidade da MO, FDN e FDNpd (trato total):

$$
\text { Dig }=100-100 \times \frac{\text { Indicador no alimento }(\% \mathrm{MS}) \times \% \text { do nutriente nas fezes }(\% \mathrm{MS})}{\text { Indicador nas fezes }(\% \mathrm{MS}) \times \% \text { do nutriente no alimento }(\% \mathrm{MS})}
$$

Ordenharam-se as vacas mecânica e diariamente às $05 \mathrm{~h} 30 \mathrm{~min}$ e $17 \mathrm{~h} 30 \mathrm{~min}$, e registrou-se a produção de leite a cada ordenha. Coletaram-se amostras de leite das ordenhas da manhã e da tarde do dia oito ao dia doze de cada período, sendo conservadas em frascos contendo 2-bromo-2nitropropane-1,3-diol, armazenadas em refrigerador, e posteriormente analisadas no Laboratório de Fisiologia da Lactação do Departamento de Zootecnia (ESALQ/USP), para determinação das concentrações de gordura e proteína, utilizando-se equipamento de espectroscopia infravermelha (Milkoscan $4000 \mathrm{Basic}^{\circledR}$ ) com princípio analítico infravermelho próximo. Calcularam-se as concentrações e produções de gordura e proteína para cada vaca e período considerando-se a média ponderada das amostras e ordenhas realizadas. Corrigiu-se a produção de leite para 3,5\% de gordura (LCG 3,5\%), segundo TYRRELL \& REID (1965), em que LCG $3,5 \%=(0,4324 \times \mathrm{kg}$ de leite/dia $)+$ $(16,216 \times \mathrm{kg}$ de gordura/dia).

Coletaram-se amostras de aproximadamente $200 \mathrm{~mL}$ de fluido ruminal de cinco pontos diferentes do rúmen com auxílio de um tubo rígido de metal de parede perfurada inserido no rúmen, através da cânula, acoplado a um dispositivo gerador de pressão negativa, do décimo ao décimo segundo dia de cada período de coleta. Coletaram-se as amostras imediatamente antes da alimentação da manhã e posteriormente em intervalos de três horas durante $\mathrm{o}$ décimo dia. A partir do décimo primeiro dia, os horários de amostragem foram adiantados em uma hora e meia, totalizando 16 horários de coleta, escalonados no tempo durante os três dias. Determinou-se o $\mathrm{pH}$ do fluido ruminal por meio de um potenciômetro digital modelo Licit $^{\circledR}$, imediatamente após as coletas, e em seguida alíquotas em duplicata de quatro $\mathrm{mL}$ do fluido ruminal foram adicionadas com um $\mathrm{mL}$ de ácido metafosfórico $25 \%$, congeladas e armazenadas em freezer a $-20^{\circ} \mathrm{C}$. Após o descongelamento, centrifugaram-se as amostras a $12.500 \times \mathrm{g}$, a $4^{\circ} \mathrm{C}$ por 15 minutos, e o sobrenadante foi analisado para determinação de ácidos graxos de cadeira curta (AGCC), conforme ERWIN et al. (1961), utilizandose cromatógrafo a gás (modelo 5890 Série II, Hewlett Packard).

Procedeu-se a análise estatística por meio do procedimento de modelos mistos (PROC MIXED) do pacote estatístico do SAS (1999), adotando-se o seguinte modelo: $Y i j k=\mu+\mathrm{Vi}+\mathrm{Pj}+\mathrm{Tk}+$ eijk, em que: Yijk corresponde ao dado referente à i-ésima vaca, ao j-ésimo período e ao k-ésimo tratamento; $\mu$ corresponde à média geral observada; Vi corresponde ao efeito aleatório da i-ésima vaca; $\mathrm{Pj}$ corresponde ao efeito fixo do j-ésimo período; Tk corresponde ao efeito fixo do k-ésimo tratamento; e eijk corresponde ao erro aleatório associado a iésima vaca, j-ésimo período e k-ésimo tratamento. Compararam-se os efeitos de tratamentos por meio dos seguintes contrastes ortogonais:

SMB vs. CAN, CAS e FA: comparação da dieta SMB (controle negativo) contendo $14 \%$ de FDNf da silagem de milho versus a inclusão de $8 \%$ de FDN de três forragens (CAN, CAS e FA) em uma dieta basal com $14 \%$ de FDNf da silagem de milho.

SMA vs. CAN, CAS e FA: comparação da dieta SMA (controle positivo) contendo $22 \%$ de FDNf da silagem de milho versus a inclusão de $8 \%$ de FDN de três forragens (CAN, CAS e FA) em uma dieta basal com 14\% de FDNf da silagem de milho.

CAN e CAS vs. FA: comparação da inclusão de $8 \%$ de FDNf da cana-de-açúcar (in natura e tratada com $\mathrm{NaOH}$ ) em uma dieta basal com $14 \%$ de FDNf da silagem de milho versus a inclusão de $8 \%$ de FDNf do feno de alfafa em uma dieta basal com $14 \%$ de FDNf da silagem de milho.

CAN vs. CAS: comparação da inclusão de 
8\% de FDNf da cana-de-açúcar in natura em uma dieta basal com 14\% de FDNf da silagem de milho versus a inclusão de $8 \%$ de FDNf da cana-de-açúcar tratada com $\mathrm{NaOH}$ em uma dieta basal com $14 \%$ de FDNf da silagem de milho.

Analisaram-se os dados de $\mathrm{pH}$ do fluido ruminal, produção total e proporção molar dos AGCC nos diferentes horários de coleta, utilizandose o procedimento de medidas repetidas no tempo (REPEATED), com estrutura de covariância autoregressiva de primeira ordem $[\mathrm{AR}(1)]$, por meio do procedimento de modelos mistos (PROC MIXED) do pacote estatístico do SAS (1999). Adotou-se nível de significância de 5\% $(\mathrm{P}<0,05)$ e tendências de $10 \%$ $(\mathrm{P}<0,10)$ entre os tratamentos. Os valores reportados nas tabelas de resultados representam as médias dos quadrados mínimos e correspondentes erros-padrão da média (EPM).

\section{RESULTADOS E DISCUSSÃO}

Devido às flutuações ocorridas nos valores da FDNf no decorrer deste experimento, as concentrações de FDNf e FDN total foram, em termos numéricos, ligeiramente inferiores às concentrações inicialmente estabelecidas de $14 \%$ de FDNf para a silagem de milho e $22 \%$ de FDN total na dieta SMB, 14\% de FDNf para a silagem de milho e $28 \%$ de FDN total nas dietas CAN, CAS e FA, e $22 \%$ de FDNf para a silagem de milho e $28 \%$ de FDN total na dieta SMA. Contrariamente, as concentrações de FDNf da cana-de-açúcar in natura, cana-de-açúcar $+\mathrm{NaOH}$, e feno de alfafa foram, também em termos numéricos, ligeiramente superiores ao objetivo inicial de incluir $8 \%$ de FDNf nas dietas CAN, CAS e FA, respectivamente (Tabela $1)$.

Não houve efeito de tratamento sobre o consumo de MS e MO (P>0,05), porém o consumo de FDN e FDNpd foram reduzidos $(\mathrm{P}<0,01)$ na dieta SMB em relação às demais dietas (Tabela 2), resultado já esperado em função da menor concentração numérica de FDN na dieta SMB (Tabela 1). O efeito da fonte da FDN sobre o consumo de MS foi avaliado em alguns experimentos, sendo que, de forma geral, não houve efeito dessa variável sobre o consumo de MS quando as dietas foram balanceadas para concentrações equivalentes de FDN total (BEAUCHEMIN, 1991; POORE et al., 1991; POORE et al., 1993). Apesar desses resultados, é preciso considerar que a importância da concentração e fonte da FDN da ração sobre o consumo de MS depende também da exigência de energia do animal (ALLEN, 2000) e da digestibilidade da FDN do alimento (OBA \& ALLEN, 1999).

TABELA 2- Efeito da substituição parcial da FDN da silagem de milho pela FDN da cana-de-açúcar ou feno de alfafa sobre o consumo e digestibilidade de nutrientes

\begin{tabular}{|c|c|c|c|c|c|c|c|c|c|c|}
\hline \multirow[b]{2}{*}{ Item } & \multicolumn{5}{|c|}{ Dietas } & \multirow[b]{2}{*}{$\mathrm{EPM}^{6}$} & \multicolumn{4}{|c|}{$\mathrm{P}$} \\
\hline & $\mathrm{SMB}^{1}$ & $\mathrm{CAN}^{2}$ & $\mathrm{CAS}^{3}$ & $\mathrm{FA}^{4}$ & $\mathrm{SMA}^{5}$ & & $\begin{array}{l}\text { SMB vs. } \\
\text { CAN, } \\
\text { CAS e FA }\end{array}$ & $\begin{array}{l}\text { SMA vs. } \\
\text { CAN, CAS e } \\
\text { FA }\end{array}$ & $\begin{array}{l}\text { CAN e } \\
\text { CAS vs. } \\
\text { FA }\end{array}$ & $\begin{array}{l}\text { CAN } \\
\text { vs. CAS }\end{array}$ \\
\hline Consumo de MS, kg/dia & 16,8 & 17,8 & 16,5 & 17,3 & 16,8 & 0,7 & NS & NS & NS & NS \\
\hline $\begin{array}{l}\text { Consumo de MO, } \\
\mathrm{kg} / \mathrm{dia}\end{array}$ & 15,7 & 16,7 & 15,3 & 16,0 & 15,6 & 0,6 & NS & NS & NS & NS \\
\hline $\begin{array}{l}\text { Consumo de FDN, } \\
\mathrm{kg} / \mathrm{dia}\end{array}$ & 3,5 & 4,9 & 4,7 & 4,8 & 4,6 & 0,19 & $<0,01$ & NS & NS & NS \\
\hline $\begin{array}{l}\text { Consumo de FDNpd, } \\
\mathrm{kg} / \text { dia }\end{array}$ & 2,9 & 3,5 & 3,5 & 3,4 & 3,5 & 0,14 & $<0,01$ & NS & NS & NS \\
\hline $\begin{array}{l}\text { Digestibilidade, \% } \\
\text { MS }\end{array}$ & 73,2 & 65,5 & 68,6 & 64,5 & 61,8 & 1,1 & $<0,01$ & $<0,01$ & 0,09 & NS \\
\hline MO & 74,6 & 67,6 & 70,2 & 66,4 & 63,8 & 1,1 & $<0,01$ & $<0,01$ & 0,09 & NS \\
\hline FDN no rúmen & 48,1 & 39,6 & 45,1 & 45,4 & 37,2 & 5,2 & NS & NS & NS & NS \\
\hline FDN no trato total & 44,7 & 34,5 & 44,9 & 46,7 & 33,9 & 3,2 & NS & NS & NS & 0,05 \\
\hline FDNpd no rúmen & 50,2 & 55,8 & 62,5 & 57,2 & 49,8 & 4,3 & NS & 0,06 & NS & NS \\
\hline FDNpd no trato total & 60,2 & 48,6 & 65,2 & 56,3 & 48,6 & 3,6 & NS & 0,06 & NS & 0,01 \\
\hline
\end{tabular}

${ }^{1} \mathrm{SMB}=$ silagem de milho baixa (14\% FDNf de silagem de milho e $22 \%$ FDN total)

${ }^{2} \mathrm{CAN}=$ cana-de-açúcar in natura $(14 \%$ FDNf de silagem de milho $+8 \%$ FDNf de cana-de-açúcar e $28 \%$ FDN total)

${ }^{3} \mathrm{CAS}=$ cana-de-açúcar $+\mathrm{NaOH}(14 \%$ FDNf de silagem de milho $+8 \%$ FDNf de cana-de-açúcar e $28 \%$ FDN total $)$

${ }^{4} \mathrm{FA}=$ feno de alfafa (14\% de FDNf de silagem de milho $+8 \%$ FDNf de feno de alfafa e $28 \%$ FDN total)

${ }^{5} \mathrm{SMA}=$ silagem de milho alta (22\% FDNf de silagem de milho e $28 \%$ FDN total)

${ }^{6} \mathrm{EPM}=$ erro padrão da média

NS = não significativo $(\mathrm{P}>0,05)$ 
A amplitude de variação da fonte e digestibilidade da FDNf no presente experimento foi, até certo ponto, limitada pela substituição parcial da FDN da silagem de milho pela FDN das outras forragens. Além disso, as dietas foram balanceadas para concentração de FDN total próxima ao limite mínimo estabelecido pelo NRC (2001) e com proporção relativamente elevada de CNF $(44,8$ a $52,1 \%$ da MS, Tabela 1), levando-se em consideração o potencial médio de produção das vacas utilizadas neste experimento $(18,2 \mathrm{~kg} / \mathrm{dia}) . \mathrm{Em}$ outras oportunidades, a substituição parcial ou total da silagem de milho pela cana-de-açúcar em maiores níveis resultou na redução do consumo de MS (CORREA et al., 2000; MAGALHÃES et al., 2004; MENDONÇA et al., 2004; COSTA et al., 2005). Além disso, a compilação dos resultados de seis experimentos comparando a alimentação exclusiva de silagem de milho ou cana-de-açúcar como fonte de forragem também revelou redução no consumo de MS (OLIVEIRA et al., 2011). A redução no consumo de MS observada em dietas com maior proporção de cana-de-açúcar é explicada pelo efeito de repleção da FDN, que tem origem na baixa taxa de digestão da FDNpd e concentração elevada da FDN $i$ (PEREIRA et al., 2000; OLIVEIRA et al., 2011).

Houve aumento $(\mathrm{P}<0,01)$ na digestibilidade da MS e MO na dieta SMB, comparada às dietas CAN, CAS e FA, o que pode ser explicado pela menor proporção de FDNf e FDN total e maior proporção de concentrado e $\mathrm{CNF}$, possivelmente resultando em maior sincronismo entre carboidratos fermentescíveis e aporte de nitrogênio.

Em se tratando da dieta SMA, houve redução $(\mathrm{P}<0,01)$ na digestibilidade da MS e MO, ausência de resposta $(\mathrm{P}>0,05)$ de tratamento sobre a digestibilidade da FDN (rúmen e trato total) e tendência $(\mathrm{P}=0,06)$ de redução na digestibilidade da FDNpd (rúmen e trato total), em comparação com as dietas CAN, CAS e FA. Em função do balanceamento das dietas SMA, CAN, CAS e FA, as quais contêm níveis bastante próximos de FDNf, FDN total e FDNpd (Tabela 1), esses resultados são de difícil explicação e contrários aos frequentemente encontrados na literatura, uma vez que existem relatos consistentes evidenciando que a digestibilidade da FDN da silagem de milho é maior que a da cana-de-açúcar, em função da menor lignificação da parede celular da planta do milho (PEREIRA et al., 2000; CAMPOS et al., 2001; MENDONÇA et al., 2004; COSTA et al., 2005; MAGALHÃES et al., 2006).

As dietas com cana-de-açúcar apresentaram tendência $(\mathrm{P}=0,09)$ para maior digestibilidade da MS e MO comparada à dieta FA, mas o tratamento com $\mathrm{NaOH}$ na dieta CAS não alterou $(\mathrm{P}>0,05)$ a digestibilidade da MS e MO, em comparação à dieta CAN.

Houve resposta do tratamento com $\mathrm{NaOH}$ na dieta CAS sobre a digestibilidade da FDN $(\mathrm{P}<0,05) \mathrm{e}$ FDNpd $(\mathrm{P}<0,01)$, ambas no trato total, em relação à dieta CAN, demonstrando um possível benefício do rompimento das ligações entre a lignina e os demais componentes da parede celular, e provavelmente aumentando a quantidade de carboidratos fibrosos para degradação realizada pelos microrganismos do rúmen (KLOPFENSTEIN \& OWEN, 1981). Entretanto, esse efeito do tratamento com $\mathrm{NaOH}$ sobre a digestibilidade da fração fibrosa da dieta CAS no trato total não se traduziu em aumento no consumo de MS (Tabela 2) e tampouco na produção de leite das vacas alimentadas com essa dieta (Tabela $3)$.

A produção de leite, produção de leite corrigida para $3,5 \%$ de gordura e composição do leite não foram afetadas $(\mathrm{P}>0,05)$ pela concentração ou fonte de FDNf das rações experimentais (Tabela 3). A ausência de resposta para concentração ou fonte de FDNf sobre a composição do leite sugere que as dietas CAN, CAS e FA apresentaram FDNe equivalente à da silagem de milho, tanto na dieta SMB (controle negativo) quanto na dieta SMA (controle positivo). Entretanto, a substituição parcial ou total da silagem de milho pela cana-de-açúcar resultou em queda na produção de leite, porém, assim como nessa pesquisa, sem efeitos significativos sobre o teor de gordura (CORREA et al., 2000; MAGALHÃES et al., 2004; MENDONÇA et al., 2004; COSTA et al., 2005). Em sua metaanálise, OLIVEIRA et al. (2011) reportaram diminuição da produção de leite de vacas alimentadas com cana-de-açúcar comparada à silagem de milho como fontes únicas de forragem.

Na Tabela 4 estão apresentados os resultados dos parâmetros ruminais. $\mathrm{O} \mathrm{pH}$ ruminal não foi alterado $(\mathrm{P}>0,05)$ pelo nível de FDNf na dieta, corroborando os dados relatados por MAGALHÃES et al. (2006), os quais não encontraram diferença no $\mathrm{pH}$ do fluido ruminal com níveis crescentes de canade-açúcar em substituição à silagem de milho na ração $(0 ; 33,3 ; 66,6$ e $100 \%$ da MS). Porém, a substituição parcial da FDN da silagem de milho pela FDN do feno de alfafa na dieta FA reduziu $(\mathrm{P}=0,03)$ o $\mathrm{pH}$ ruminal em relação às dietas $\mathrm{CAN}$ e CAS. Para as dietas com $22 \%$ de FDNf, o pH ruminal se manteve sempre acima de 6,0; mas na dieta SMB o pH ruminal esteve inferior a 6,0 por até duas horas durante o dia.

Ci. Anim. Bras., Goiânia, v.13, n.4, p. 450-459, out./dez. 2012 
TABELA 3- Efeito da substituição parcial da FDN da silagem de milho pela FDN da cana-de-açúcar ou feno de alfafa sobre a produção e composição do leite

\begin{tabular}{lcccccc}
\hline Item & $\mathrm{SMB}^{1}$ & $\mathrm{CAN}^{2}$ & $\mathrm{CAS}^{3}$ & $\mathrm{FA}^{4}$ & $\mathrm{SMA}^{5}$ & $\mathrm{EPM}^{6}$ \\
\hline Leite, $\mathrm{kg} / \mathrm{dia}$ & 18,6 & 17,8 & 18,4 & 18,0 & 18,4 & 1,1 \\
LCG 3,5\%, kg/dia & 18,7 & 18,6 & 19,0 & 17,8 & 18,8 & 1,0 \\
Gordura, \% & 3,6 & 3,7 & 3,7 & 3,4 & 3,6 & 0,17 \\
Gordura, $\mathrm{kg} / \mathrm{dia}$ & 0,66 & 0,67 & 0,68 & 0,62 & 0,67 & 0,04 \\
Proteína, \% & 3,4 & 3,4 & 3,3 & 3,4 & 3,3 & 0,12 \\
Proteína, $\mathrm{kg} / \mathrm{dia}$ & 0,63 & 0,62 & 0,61 & 0,61 & 0,60 & 0,038 \\
\hline
\end{tabular}

${ }^{1} \mathrm{SMB}=$ silagem de milho baixa (14\% FDNf de silagem de milho e $22 \%$ FDN total)

${ }^{2} \mathrm{CAN}=$ cana-de-açúcar in natura $(14 \%$ FDNf de silagem de milho $+8 \%$ FDNf de cana-de-açúcar e $28 \%$ FDN total $)$

${ }^{3} \mathrm{CAS}=$ cana-de-açúcar $+\mathrm{NaOH}(14 \%$ FDNf de silagem de milho $+8 \%$ FDNf de cana-de-açúcar e $28 \%$ FDN total $)$

${ }^{4} \mathrm{FA}=$ feno de alfafa (14\% de FDNf de silagem de milho + $8 \%$ FDNf de feno de alfafa e $28 \%$ FDN total)

${ }^{5} \mathrm{SMA}=$ silagem de milho alta (22\% FDNf de silagem de milho e $28 \%$ FDN total)

${ }^{6} \mathrm{EPM}=$ erro padrão da média

${ }^{7} \mathrm{LCG} 3,5 \%, \mathrm{~kg} / \mathrm{dia}=$ leite corrigido para 3,5\% de gordura (TYRREL \& REID, 1965)

TABELA 4- Efeito da substituição parcial da FDN da silagem de milho pela FDN da cana-de-açúcar ou feno de alfafa sobre os parâmetros ruminais

\begin{tabular}{|c|c|c|c|c|c|c|c|c|c|c|}
\hline \multirow[b]{2}{*}{ Item } & \multicolumn{5}{|c|}{ Dietas } & \multirow[b]{2}{*}{$\mathrm{EPM}^{6}$} & \multicolumn{4}{|c|}{$\mathrm{P}$} \\
\hline & $\mathrm{SMB}^{1}$ & $\mathrm{CAN}^{2}$ & $\mathrm{CAS}^{3}$ & $\mathrm{FA}^{4}$ & $\mathrm{SMA}^{5}$ & & $\begin{array}{c}\text { SMB vs. } \\
\text { CAN, } \\
\text { CAS e FA }\end{array}$ & $\begin{array}{c}\text { SMA vs. } \\
\text { CAN, CAS } \\
\text { e FA }\end{array}$ & $\begin{array}{c}\text { CAN e } \\
\text { CAS vs. } \\
\text { FA }\end{array}$ & $\begin{array}{c}\text { CAN vs. } \\
\text { CAS }\end{array}$ \\
\hline $\mathrm{pH}$ & 6,24 & 6,35 & 6,38 & 6,23 & 6,27 & 0,17 & $\mathrm{NS}$ & NS & 0,03 & NS \\
\hline $\begin{array}{l}\mathrm{AGCC}^{7}, \mathrm{~m} M \\
\mathrm{AGCC}, \mathrm{mol} / 100 \mathrm{~mol}\end{array}$ & 122 & 123 & 115 & 128 & 122 & 6,0 & NS & NS & NS & NS \\
\hline Acetato & 60,9 & 64,1 & 65,0 & 62,2 & 59,7 & 1,6 & 0,01 & $<0,01$ & 0,08 & NS \\
\hline Propionato & 23,8 & 21,9 & 20,6 & 23,9 & 25,1 & 1,5 & NS & $<0,01$ & 0,01 & 0,07 \\
\hline Butirato & 11,4 & 10,5 & 10,9 & 10,5 & 11,3 & 0,4 & $<0,01$ & $<0,01$ & NS & NS \\
\hline Acetato:pro-pionato & 2,71 & 2,94 & 3,23 & 2,72 & 2,47 & 0,23 & 0,07 & $<0,01$ & 0,02 & 0,05 \\
\hline
\end{tabular}

${ }^{\mathrm{T}} \mathrm{SMB}=$ silagem de milho baixa (14\% FDNf de silagem de milho e $22 \%$ FDN total)

${ }^{2} \mathrm{CAN}=$ cana-de-açúcar in natura $(14 \%$ FDNf de silagem de milho $+8 \%$ FDNf de cana-de-açúcar e $28 \%$ FDN total)

${ }^{3} \mathrm{CAS}=$ cana-de-açúcar $+\mathrm{NaOH}(14 \%$ FDNf de silagem de milho $+8 \%$ FDNf de cana-de-açúcar e $28 \%$ FDN total $)$

${ }^{4} \mathrm{FA}=$ feno de alfafa (14\% de FDNf de silagem de milho $+8 \%$ FDNf de feno de alfafa e $28 \%$ FDN total)

${ }^{5} \mathrm{SMA}=$ silagem de milho alta (22\% FDNf de silagem de milho e $28 \%$ FDN total)

${ }^{6} \mathrm{EPM}=$ erro padrão da média

${ }^{7}$ Ácidos graxos de cadeia curta

$\mathrm{NS}=$ não significativo $(\mathrm{P}>0,05)$

$\mathrm{O} \mathrm{pH}$ ruminal inferior a seis (6) tem sido associado à redução na digestão da FDN (MERTENS, 1979). O decréscimo do $\mathrm{pH}$ ruminal na dieta SMB pode estar associado à maior proporção de concentrados e carboidratos fermentescíveis, comparado às dietas com $22 \%$ de FDNf. Além disso, alterações no padrão de consumo de MS e atividade de ruminação podem ter determinado a queda do $\mathrm{pH}$ ruminal por um curto intervalo de tempo. Entretanto, a queda do $\mathrm{pH}$ ruminal observada na dieta SMB não foi de magnitude suficiente para alterar a digestão da FDN.

Embora a concentração total dos AGCC não tenha sido afetada pelos tratamentos, as proporções molares dos AGCC e a relação acetato:propionato foram alteradas pelo nível e fonte de FDNf na ração (Tabela 4). Na dieta SMB houve tendência $(\mathrm{P}=0,07)$ de redução da relação acetato:propionato em decorrência da menor $(\mathrm{P}=0,01)$ proporção molar de acetato, comparada às dietas CAN, CAS e FA. Na dieta SMA, tanto a redução $(\mathrm{P}<0,01)$ na proporção molar de acetato, quanto o aumento $(\mathrm{P}<0,01)$ na proporção molar de propionato ocasionaram redução $(\mathrm{P}<0,01)$ na relação acetato:propionato, comparada às dietas CAN, CAS e FA. Houve tendência $(\mathrm{P}=0,08)$ de aumento na proporção molar de acetato nas dietas CAN e CAS em relação à dieta $\mathrm{FA}$, e aumento $(\mathrm{P}=0,01)$ na proporção molar de propionato 
na dieta $\mathrm{FA}$, comparada às dietas $\mathrm{CAN}$ e CAS, resultando no aumento $(\mathrm{P}=0,02)$ da relação acetato:propionato nas dietas CAN e CAS, em comparação à dieta FA. O tratamento da cana-deaçúcar com $\mathrm{NaOH}$ resultou em tendência $(\mathrm{P}=0,07)$ de redução na proporção molar de propionato e aumento $(\mathrm{P}=0,05)$ na relação acetato:propionato na dieta $\mathrm{CAS}$, comparada à dieta $\mathrm{CAN}$.

As proporções molares de butirato também foram alteradas pelos tratamentos. Nesse caso, as dietas com silagem de milho como única fonte de FDNf aumentaram $(\mathrm{P}<0,01)$ a proporção molar de butirato, comparadas às dietas CAN, CAS e FA.

Variações na produção e proporções molares de AGCC são determinadas principalmente pelo substrato fermentado e $\mathrm{pH}$ do fluido ruminal (RUSSELL, 1998). Assim, variações na concentração e composição dos CNF e diferenças na capacidade de troca de cátions (CTC) entre forragens podem ter sido responsáveis pelas alterações observadas nos padrões de fermentação ruminal. Não se avaliou o papel da CTC das forragens nesse experimento por não ser o objetivo deste trabalho. Apesar dos efeitos significativos de tratamento sobre as relações acetato:propionato, os valores médios dessa relação sempre estiveram acima de dois (2) em todas as dietas e horários de coleta, considerado como limite mínimo para que não ocorram alterações no teor de gordura no leite (ERDMAN, 1988).

\section{CONCLUSÕES}

Apesar das variações observadas no padrão de fermentação ruminal e na digestibilidade das dietas, o desempenho e o teor de gordura no leite não foram alterados pela substituição parcial da FDN da silagem de milho pela FDN de outras forragens. O tratamento da cana-de-açúcar com $\mathrm{NaOH}$ não trouxe benefícios em relação à ração com cana-de-açúcar in natura. Assim, recomenda-se a substituição de $8 \%$ da FDN da silagem de milho pela FDN da cana-deaçúcar in natura na alimentação de vacas leiteiras com produção média de 18,2 kg de leite/dia.

\section{REFERÊNCIAS}

ALLEN, M.S. Effects of diet on short-term regulation of feed intake by lactating dairy cattle. Journal of Dairy Science, v.83, n.7, p.1598-1624, 2000.

ASSOCIATION OF OFFICIAL ANALYTICAL CHEMISTS - AOAC. Official methods of analysis. $17^{\mathrm{a}}$ edição. AOAC International, Gaithersburg, MD, 2000. 1298p.

BAL, M.A.; SHAVER, R.D.; JIROVEC, A.G.; SHINNERS, K.J.; COORS, J.G. Crop processing and chop length of corn silage: effects on intake, digestion, and milk production by dairy cows. Journal of Dairy Science, v.83, n.6, p.1264-1273, 2000.

BEAUCHEMIN, K.A. Effects of dietary neutral detergent fiber concentration and alfalfa hay quality on chewing, rumen function, and milk production of dairy cows. Journal of Dairy Science, v.74, n.9, p.3140-3151, 1991.

BERCHIELLI, T.T.; ANDRADE, P.; FURLAN, C.L. Avaliação de indicadores internos em ensaios de digestibilidade. Revista Brasileira de Zootecnia, v.29, n.3, p.830-833, 2000.

BERGER, L.; KLOPFENSTEIN, T.; BRITTON, R. Effect of sodium hydroxide on efficiency of rumen digestion. Journal of Animal Science, v.49, n.5, p.1317-1323, 1979.

CAMPOS, F.P.; SAMPAIO, A.A.M.; VIEIRA, P.F.; BOSE, M.L.V. Digestibilidade in vitro/gás de volumosos exclusivos ou combinados avaliados pelo resíduo remanescente da digestão da matéria seca e produção de gás. Revista Brasileira de Zootecnia, v.30, n.5, p.15791589, 2001.

CLARK, P.W.; ARMENTANO, L.E. Influence of particle size on the effectiveness of the fiber in corn silage. Journal of Dairy Science, v.82, n.3, p.581-588, 1999.

CORREA, C.E.S.; PEREIRA, M.N.; RAMOS, M.H.; OLIVEIRA, S.G.; OTA, M. Performance of dairy cows fed corn silage differing in kernel texture or sugarcane as the dietary forage. Journal of Dairy Science, v.83, suplemento 1, p.563-569, 2000.

COSTA, M.G.; CAMPOS, J.M.S.; VALADARES FILHO, S.C.; VALADARES, R.F.D.; MENDONÇA, S.S.; SOUZA, D.P.; TEIXEIRA, M.P. Desempenho produtivo de vacas leiteiras alimentadas com diferentes proporções de cana-de-açúcar e concentrado ou silagem de milho na dieta. Revista Brasileira de Zootecnia, v.34, n.6, p.2437-2445, 2005 (suplemento).

ERDMAN, R.A. Dietary buffering requirements of the lactating dairy cow: a review. Journal of Dairy Science, v.71, n.12, p.3246-3266, 1988.

ERWIN, E.S.; MARCO, G.J.; EMERY, E.M. Volatile fatty acid analyses of blood and rumen fluid by gas chromatography. Journal of Dairy Science, v.44, n.9, p.1768-1771, 1961.

KLOPFENSTEIN, T.; OWEN, F.G. Value and potencial use of crop residues and by-products in dairy rations. Journal of Dairy Science, v.64, n.6, p.1250-1268, 1981.

MAGALHÃES, A.L.R.; CAMPOS, J.M.S.; CABRAL, L.S.; MELLO, R.; FREITAS, J.A.; TORRES, R.A.; VALADARES FILHO, S.C.; ASSIS, A.J. Cana-de-açúcar em substituição à silagem de milho em dietas para vacas em lactação: parâmetros digestivos e ruminais. Revista Brasileira de Zootecnia, v.35, n.2, p.591-599, 2006.

MAGALHÃES, A.L.R.; CAMPOS, J.M.S.; VALADARES FILHO, S.C.; TORRES, R.A; MENDES NETO, J.; ASSIS, A.J. Cana-de-açúcar em substituição à 
silagem de milho em dietas para vacas em lactação: desempenho e viabilidade econômica. Revista Brasileira de Zootecnia, v.33, n.5, p.1292-1302, 2004.

MENDONÇA, S.S.; CAMPOS, J.M.S.; VALADARES FILHO, S.C.; VALADARES, R.F.D.; SOARES, C.A.; LANA, R.P.; QUEIROZ, A.C.; ASSIS, A.J.; PEREIRA, M.L.A. Consumo, digestibilidade aparente, produção e composição do leite e variáveis ruminais em vacas leiteiras alimentadas com dietas à base de cana-de-açúcar. Revista Brasileira de Zootecnia, v.33, n.2, p.481-492, 2004

MERTENS, D.R. Creating a system for meeting the fiber requirements of dairy cows. Journal of Dairy Science, v.80, n.7, p.1463-1481, 1997.

MERTENS, D.R. Effects of buffers upon fiber digestion. Piscataway, NJ: Church and Dwight Co., Inc., 1979. $138 \mathrm{p}$.

MOONEY, C.S.; ALLEN, M.S. Physical effectiveness of the neutral detergent fiber of whole linted cottonseed relative to that of alfalfa silage at two lengths of cut. Journal of Dairy Science, v.80, n.9, p.2052-2061, 1997.

NATIONAL RESEARCH COUNCIL - NRC. Nutrient requirements of dairy cattle. $7^{\mathrm{a}}$ edição revisada. National Academy of Sciences, Washington, DC, 2001. 381p.

OBA, M.; ALLEN, M.S. Evaluation of the importance of the digestibility of neutral detergent fiber from forage: effects on dry matter intake and milk of dairy cows. Journal of Dairy Science, v.82, n.3, p.589-596, 1999.

OLIVEIRA, A.S.; DETMANN, E.; CAMPOS, J.M.S.; PINA, D.S.; SOUZA, S.M.; COSTA, M.G. Meta-análise do impacto da fibra em detergente neutro sobre o consumo, a digestibilidade e o desempenho de vacas leiteiras em lactação. Revista Brasileira de Zootecnia, v.40, n.7, p.1587-1595, 2011.

PEREIRA, E.S.; QUEIROZ, A.C.; PAULINO, M.F.; CECON, P.R.; VALADARES FILHO, S.C.; MIRANDA, L.F.; FERNANDES, A.M.; CABRAL, L.S. Determinação das frações proteicas e de carboidratos e taxas de degradação in vitro da cana-de-açúcar, da cama de frango e do farelo de algodão. Revista Brasileira de Zootecnia, v.29, n.6, p.1887-1893, 2000.

POORE, M.H.; MOORE, J.A.; SWINGLE, R.S.; ECK, T.P.; BROWN, W.H. Response of lactating Holstein cows to diets varying in fiber source and ruminal starch degradability. Journal of Dairy Science, v.76, n.8, p.2235-2243, 1993.

POORE, M.H.; MOORE, J.A.; SWINGLE, R.S.; ECK, T.P.; BROWN, W.H. Wheat straw or alfalfa hay in diets with $30 \%$ neutral detergent fiber for lactating Holstein cows. Journal of Dairy Science, v.74, n.9, p.3152-3159, 1991.

RUSSELL, J.B. The importance of $\mathrm{pH}$ in the regulation of ruminal acetate to propionate ratio and methane production in vitro. Journal of Dairy Science, v.81, n.12, p.3222-3230, 1998.

SAS Institute. SAS/STAT User's Guide: Statistics, Version 9.2 Edition. SAS Inst. Inc., Cary, NC, 1999.

SCHNEIDER, B.H.; FLATT, W.P. The evaluation of feeds through digestibility experiments. Athens: The University of Georgia Press, 1975. 423p.

SCHWAB, E.C.; SHAVER, R.D.; SHINNERS, K.J.; LAUER, J.G.; COORS, J.G. Processing and chop length effects in brown-midrib corn silage on intake, digestion, and milk production by dairy cows. Journal of Dairy Science, v.85, n.3, p.613-623, 2002.

SOITA, H.W.; CHRISTENSEN, D.A.; McKINNON, J.J. Influence of particle size on the effectiveness of the fiber in barley silage. Journal of Dairy Science, v.83, n.10, p.2295-2300, 2000.

TYRRELL, H.F.; REID, J.T. Prediction of the energy value of cow's milk. Journal of Dairy Science, v.48, n.9, p.1215-1223, 1965.

VAN SOEST, P.J.; ROBERTSON, J.B.; LEWIS, B.A. Methods for dietary fiber, neutral detergent fiber, and nonstarch polysaccharides in relation to animal nutrition. Journal of Dairy Science, v.74, n.10, p.35833597, 1991. 